OPEN ACCESS

Edited by:

Yi-Wei Tang,

Cepheid, United States

Reviewed by:

Bryan Schmitt,

Indiana University Bloomington,

United States

Xinxin Lu,

Capital Medical University, China

${ }^{*}$ Correspondence:

Li Zhang

zhangli19870711@163.com

Ying-Chun Xu

xycpumch@139.com

${ }^{\dagger}$ These authors have contributed equally to this work and share first authorship

Specialty section: This article was submitted to Clinical Microbiology, a section of the journal Frontiers in Cellular and Infection Microbiology

Received: 29 March 2021 Accepted: 14 June 2021 Published: 06 July 2021

Citation: Ning $Y-T$, Yang $W-H$, Zhang $W$, Xiao $M$, Wang $Y$, Zhang J-J, Zhang $G$, Duan S-M, Dong A-Y, Guo D-W

Zou G-L, Wen H-N, Guo Y-Y, Chen L-P, Chai M, He J-D, Duan Q,

Zhang L-X, Zhang L and Xu Y-C (2021) Developing Two Rapid Protein Extraction Methods Using Focused-

Ultrasonication and Zirconia-Silica Beads for Filamentous Fung Identification by MALDI-TOF MS. Front. Cell. Infect. Microbiol. 11:687240. doi: 10.3389/fcimb.2021.687240

\section{Developing Two Rapid Protein Extraction Methods Using Focused- Ultrasonication and Zirconia-Silica Beads for Filamentous Fungi Identification by MALDI-TOF MS}

\author{
Ya-Ting Ning ${ }^{1,2,3 t}$, Wen-Hang Yang ${ }^{1,2,3 t}$, Wei Zhang ${ }^{2,4}$, Meng Xiao ${ }^{1,2,3}$, Yao Wang ${ }^{1,3}$, \\ Jing-Jia Zhang ${ }^{1,3}$, Ge Zhang ${ }^{1,3}$, Si-Meng Duan ${ }^{1,3}$, Ai-Ying Dong ${ }^{5}$, Da-Wen Guo ${ }^{6}$, \\ Gui-Ling Zou ${ }^{7}$, Hai-Nan Wen ${ }^{8}$, Yan-Yan Guo ${ }^{9}$, Li-Ping Chen ${ }^{10}$, Miao Chai ${ }^{11}$, \\ Jing-Dong He ${ }^{12}$, Qiong Duan ${ }^{13}$, Li-Xia Zhang ${ }^{14}$, Li Zhang ${ }^{1,3^{*}}$ and Ying-Chun $\mathrm{Xu}^{1,3 *}$ \\ ${ }^{1}$ Department of Clinical Laboratory, State Key Laboratory of Complex Severe and Rare Diseases, Peking Union Medical \\ College Hospital, Chinese Academy of Medical Sciences and Peking Union Medical College, Beijing, China, ${ }^{2}$ Graduate \\ School, Chinese Academy of Medical Sciences and Peking Union Medical College, Beijing, China, ${ }^{3}$ Beijing Key Laboratory \\ for Mechanisms Research and Precision Diagnosis of Invasive Fungal Diseases, Beijing, China, ${ }^{4}$ Clinical Microbiology \\ Laboratory, The First Affiliated Hospital of Hebei North University, Zhangjiakou, China, ${ }^{5}$ Department of Clinical Laboratory, \\ North China University of Science and Technology Affiliated Hospital, Tangshan, China, ${ }^{6}$ Department of Clinical Laboratory, \\ The First Affiliated Hospital of Harbin Medical University, Harbin, China, ${ }^{7}$ Department of Clinical Laboratory, The Fourth \\ Affiliated Hospital of Harbin Medical University, Harbin, China, ${ }^{8}$ Department of Laboratory, The Affiliated Hospital of Chengde \\ Medical University, Chengde, China, ${ }^{9}$ Department of Clinical Laboratory, Tangshan Worker's Hospital, Tangshan, China, \\ 10 Department of Laboratory Medicine, Mudanijang First People's Hospital, Heilongijiang. China, ${ }^{11}$ Department of Clinical \\ Laboratory, The First Hospital of Harbin, Harbin, China, ${ }^{12}$ Department of Clinical Laboratory, Tianjin Chest Hospital, Tianjin, \\ China, ${ }^{13}$ Department of Clinical Laboratory, Jinling Province People's Hospital, Jinling, China, ${ }^{14}$ Department of Clinical \\ Laboratory, Shanxi Provincial People's Hospital, Taiyuan, China
}

Filamentous fungi identification by Matrix-assisted laser desorption ionization time-of-flight mass spectrometry (MALDI-TOF MS) has been challenging due to the lack of simple and rapid protein extraction methods and insufficient species coverage in the database. In this study, we created two rapid protein extraction methods for filamentous fungi: a one-step zirconia-silica beads method (ZSB) and a focused-ultrasonication method (FUS). The identification accuracy of two methods were evaluated with the VITEK MS, as well as number of spectra peaks and signal-to-noise ratio (S/N) with M-Discover $100 \mathrm{MALDI-TOF}$ MS compared to the routine method. The better method was applied to build a filamentous fungi in-house spectra library for the M-Discover $100 \mathrm{MS}$, and then another one and routine method were performed in parallel to verify the accuracy and commonality of the in-house library. Using the two optimized methods, the dedicated operating time before MALDI-TOF MS analysis was reduced from 30 min to 7 (ZSB) or 5 (FUS) min per sample, with only a few seconds added for each additional strain. And both two methods identified isolates from most mold types equal to or better than the routine method, and the total correct identification rate using VITEK MS was 79.67, 76.42, and 76.42\%, respectively. On the other hand, the two rapid methods generally achieved higher maximum and minimum $\mathrm{S} / \mathrm{N}$ ratios with these isolates tested as compared to the 
routine method. Besides, the ZSB method produced overall mean of maximum and minimum S/N ratio higher than that by FUS. An in-house library of M-Discover MS was successfully built from 135 isolates from 42 species belonging to 18 genera using the ZSB method. Analysis of 467 isolates resulted in $97.22 \%$ correctly identified isolates to the species level by the ZSB method versus $95.50 \%$ by the routine method. The two novel methods are time- and cost-effective and allow efficient identification of filamentous fungi while providing a simplified procedure to build an in-house library. Thus, more clinical laboratories may consider adopting MALDI-TOF MS for filamentous fungi identification in the future.

Keywords: filamentous fungi, MALDI-TOF MS, protein extraction, sample processing, zirconia-silica beads, focused-ultrasonication, in-house library

\section{INTRODUCTION}

In recent years, fungi have come to pose a serious threat to immunocompromised patients with leukemia, AIDS, or receiving chemotherapy intervention, etc. (Enoch et al., 2006; Benedict et al., 2017; Bongomin et al., 2017). Even though Candida remains the leading invasive fungi pathogen, infections due to filamentous fungi are gradually rising with high mortality (Enoch et al., 2006; Liao et al., 2013; Benedict et al., 2017; Bongomin et al., 2017). However, appropriate treatment often varies by species, thus making rapid identification essential for accurate diagnosis and better outcomes (Brown et al., 2012). Conventional identification methods of filamentous fungi based on morphological traits are time-consuming and require extensive expertise training (Larone, 2011). Moreover, less common or nonsporulating molds are difficult to identify, and phylogenetically related species with similar morphological features are challenging to discriminate (Kozel and Wickes, 2014; Li et al., 2017; Luethy and Zelazny, 2018; Wickes and Wiederhold, 2018). Molecular identification is the gold standard method to identify the above strains, but is relatively expensive and requires specialized equipment which limits its routine use in clinical laboratories (Balajee et al., 2007; Samson et al., 2014; Wickes and Wiederhold, 2018).

Matrix-assisted laser desorption/ionization-time of flight mass spectrometry (MALDI-TOF MS) has emerged as a costeffective and rapid alternative for mycobacterial, bacterial, and yeast identification (Seng et al., 2009; Fraser et al., 2016; Rodriguez-Sanchez et al., 2016). Currently, its use for the identification of filamentous fungi has gradually begun to be implemented in clinical microbiology laboratories, but has been hampered by commercial databases with limited coverage of filamentous fungi taxa and challenges of protein extraction to obtain good quality mass spectra for analysis (Welham et al., 2000; Cassagne et al., 2016; Santos et al., 2017). Building an inhouse database that contains local or less common isolates is the most optimal way to overcome the deficiencies of commercial databases (Zvezdanova et al., 2019). At present, several in-house databases in the Bruker MALDI Biotyper (Bruker Daltonics, Germany) have been developed by laboratories, significantly increasing species-assignment of filamentous fungi (De Carolis et al., 2012; Becker et al., 2014; Gautier et al., 2014; Schulthess et al., 2014; Luethy and Zelazny, 2018; Zvezdanova et al., 2019).

Protein extraction is the most critical step of filamentous fungi identification by MALDI-TOF MS, but this process faces challenges due to the robust chitinous cell wall of filamentous fungi and necessitating protein extraction via a process usually initialized by cell homogenization (Shapaval et al., 2017; Krishnaswamy et al., 2019). Moreover, routine protein extraction methods in the manufacturer's instructions and laboratory-developed procedures involve multiple steps (i.e. wash, inactivation, chemical extraction) and require $30 \mathrm{~min}$ to over an hour to perform (Chakrabarti et al., 2015; Cassagne et al., 2016). Thus, a simpler and more rapid procedure for protein extraction is urgently needed to permit routine use of MALDITOF MS for filamentous fungi identification in clinical laboratories. Ultrasound disruption is a common mechanical cell homogenization method based on high shear force, applied successfully in MALDI-TOF MS for mycobacterial identification and LC-MS/MS (Klimek-Ochab et al., 2011; Adams et al., 2016). Adaptive focused acoustics via concentrated bursts of higherfrequency ultrasonic energy allows for rapid disruption of the cell wall and concomitant protein extraction into the extraction solution within minutes (Li et al., 2015; Adams et al., 2016). Another mechanical method is bead milling, such as through the use of zirconia-silica beads and zirconium beads (Krishnaswamy et al., 2019). Proteins are released by the action of circulating beads dispersed in the fluid (Doucha and Livansky, 2008; Klimek-Ochab et al., 2011).

To simplify and expedite the sample processing before the identification of filamentous fungi isolates by MALDI-TOF MS, we created two rapid protein extraction procedures: the one-step zirconia-silica beads (ZSB) method and the focusedultrasonication method (FUS). In this study, we investigated the identification accuracy of two rapid sample processing methods in the VITEK MALDI-TOF MS system (bioMérieux, Marcy-l'Étoile, France), as well as number of mass peaks and signal-to-noise (S/N) ratio in M-Discover 100 MALDI-TOF MS (Zhuhai Meihua Medical Technology Co., Ltd., China) versus the routine method. Then according to the results, applied the better method as a means to build a filamentous fungi in-house spectra library for the M-Discover 100 MS. In addition, we evaluated the accuracy and 
commonality of the in-house library using the new method consisting of building the database and the routine method.

\section{METHODS AND MATERIALS}

\section{Isolates and Species Identification}

A total of 602 non-duplicate mold isolates recovered from various clinical specimens of patients were under the China Hospital Invasive Fungal Surveillance Net-North China Program. Isolates were cultured on Sabouraud Dextrose Agar (SDA) plates (Becton Dickinson Microbiology Systems, Sparks, MD, USA) and incubated at $28^{\circ} \mathrm{C}$ for 2 to 5 days, and mycelia were collected for genomic DNA extraction. The internal transcribed spacer region was carried out as the primary sequencing gene for species level identification (Zvezdanova et al., 2019). The $\beta$-tubulin gene was employed additionally for the Scedosporium/Pseudallescheria spp., as well as the translation elongation factor $1-\alpha$ gene for the Fusarium spp. (Gilgado et al., 2008; Wang et al., 2011). Sequencing data was analyzed using the National Center for Biotechnology Information (NCBI) or Mycobank database, and the results were accepted if homology $>98 \%$ with $>95 \%$ query coverage. One hundred twenty-three isolates belonging to 13 mold genera and 29 species were analyzed by VITEK MS to evaluate three protein extracting methods (Table 1). Another 135 clinical isolates were included in the in-house library of M-Discover 100

TABLE 1 | Identification of 123 clinical filamentous fungi isolates by VITEK MS using the routine method in comparison with two rapid methods.

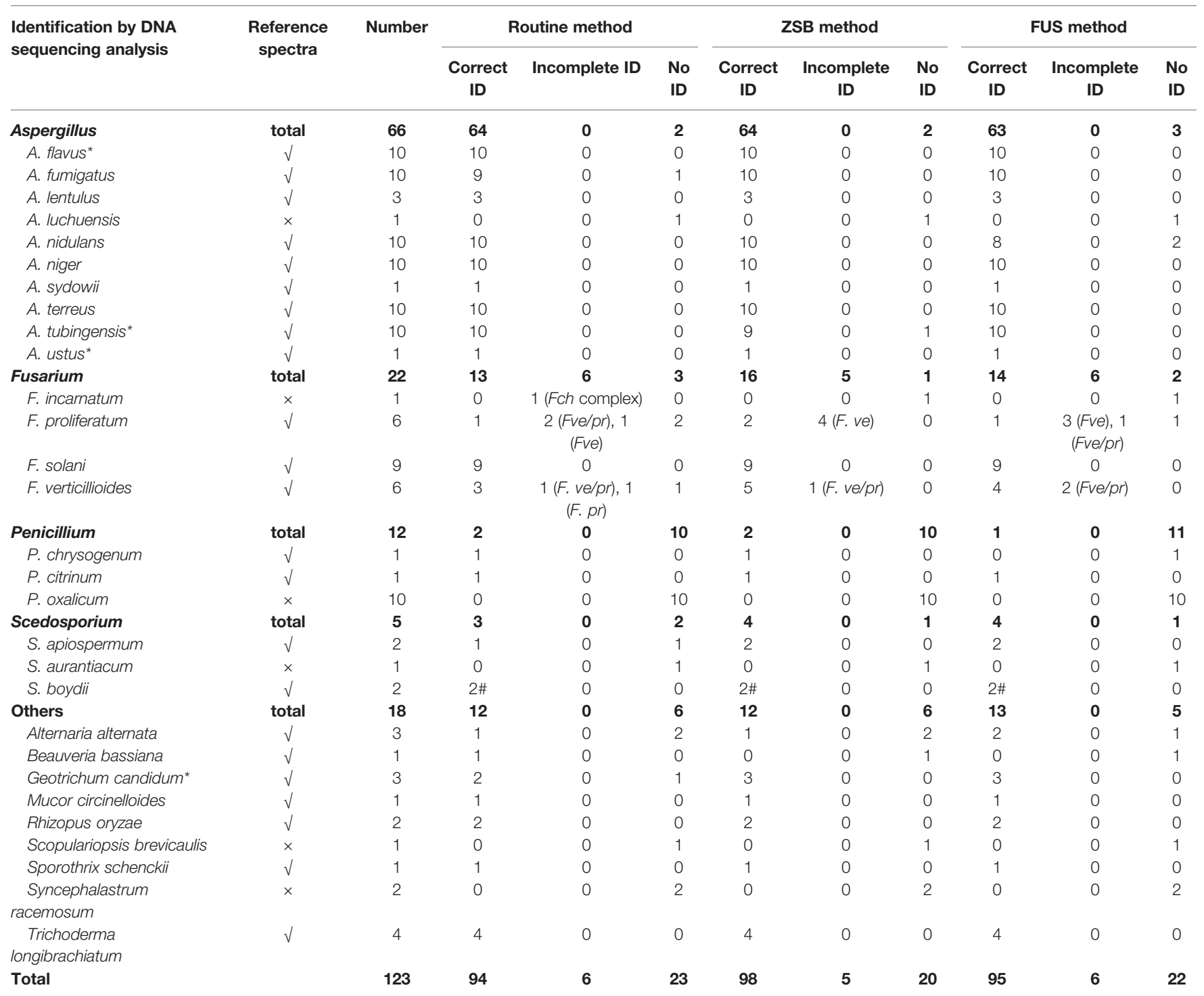

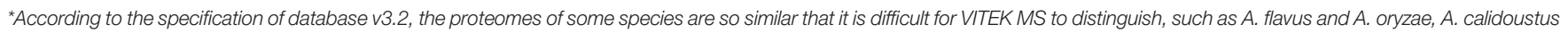
and A. ustus, Geotrichum candidum and Geotrichum klebahnii, as well as A. tubingensis which shows "A. niger complex". Those results were all considered as "correct-ID". \# VITEK MS identified S. boydii (Pseudallescheria boydii's asexual stage) as Pseudallescheria boydii. These results were considered as correct.

ID, identification; Fch, F. chlamydosporum; Fve, F. verticillioides; Fpr, F. proliferatum; Fve/pr, F. verticillioides/proliferatum. 
TABLE 2 | List of isolates included in the in-house library of M-Discover 100 MS.

\begin{tabular}{|c|c|}
\hline Identification by DNA sequencing analysis & Number of isolates \\
\hline \multicolumn{2}{|l|}{ Aspergillus } \\
\hline A. fumigatus & 15 \\
\hline A. insuetus & 2 \\
\hline A. japonicus & 1 \\
\hline A. lentulus & 1 \\
\hline A. luchuensis & 3 \\
\hline A. nidulans & 10 \\
\hline A. niger & 15 \\
\hline A. oryzae & 4 \\
\hline A. pseudoglaucus & 1 \\
\hline A. ruber & 1 \\
\hline A. sydowii & 8 \\
\hline A. tamarii & 3 \\
\hline A. terreus & 12 \\
\hline A. tubingensis & 15 \\
\hline A. uvarum & 1 \\
\hline \multicolumn{2}{|l|}{ Penicillium } \\
\hline P. chermesinum & 1 \\
\hline P. citrinum & 2 \\
\hline P. oxalicum & 7 \\
\hline \multicolumn{2}{|l|}{ Scedosporium } \\
\hline S. apiospermum & 1 \\
\hline S. aurantiacum & 1 \\
\hline S. boydii & 1 \\
\hline \multicolumn{2}{|l|}{ Trichoderma } \\
\hline T. longibrachiatum & 1 \\
\hline T. asahii & 2 \\
\hline T. coremiiforme & 1 \\
\hline T. japonicum & 1 \\
\hline \multicolumn{2}{|l|}{ Others } \\
\hline Alternaria alternata & 1 \\
\hline Arthrinium spp. & 1 \\
\hline Beauveria bassiana & 1 \\
\hline Doratomyces spp. & 1 \\
\hline Exophiala dermatitidis & 2 \\
\hline Geotrichum candidum & 2 \\
\hline Monascus purpureus & 1 \\
\hline Mucor circinelloides & 3 \\
\hline Paecilomyces variotii & 1 \\
\hline Phanerochaete chrysosporium & 1 \\
\hline Rhizomucor pusillus & 1 \\
\hline Rhizopus microsporus & 4 \\
\hline Rhizopus oryzae & 1 \\
\hline Scopulariopsis spp. & 1 \\
\hline Syncephalastrum racemosum & 2 \\
\hline Talaromyces funiculosus & 1 \\
\hline Talaromyces stollii & 1 \\
\hline Total & 135 \\
\hline
\end{tabular}

MS (Table 2); the remaining 467 isolates were analyzed using the in-house library coupled with the rapid method and routine method (Table 3).

\section{Protein Extraction}

\section{The Routine Three-Step Method}

Isolates were inoculated on SDA plates at $28^{\circ} \mathrm{C}$ for 3 days. The routine method for protein preparation was performed according to the manufacturer's instructions as described by $\mathrm{Li}$ et al. (Li et al., 2017). Briefly, one to two colonies ( $\mathrm{OD}_{600}$ of 2.0$)$ were mixed with $900 \mu \mathrm{l}$ ethanol and $300 \mu \mathrm{l}$ distilled water, followed by centrifugation for $3 \mathrm{~min}$ at $13,800 \mathrm{~g}$. The pellet was dried at room temperature (RT) for $5 \mathrm{~min}$, and then re-suspended in 50-80 $\mu \mathrm{l}$ of $70 \%$ formic acid (FA). After an incubation of $5 \mathrm{~min}$ at RT, an equal volume of acetonitrile was added. Samples were incubated again at RT for $5 \mathrm{~min}$ and subsequently centrifuged at 13,800 $\mathrm{g}$ for $1 \mathrm{~min}$.

\section{One-Step Zirconia-Silica Beads Method (ZSB)}

Rapid extraction of protein using the ZSB method was performed using the same cultures as the routine method. Approximately $1-2 \mathrm{~cm}^{2}$ pieces of mold were removed from the agar and added to a $1.5 \mathrm{ml}$ tube containing $30 \mu \mathrm{l}$ of zirconia-silica beads with a diameter of $0.5 \mathrm{~mm}$ and $60 \mathrm{ul}$ extraction solution (consisting of $30 \mathrm{ul}$ acetonitrile and $30 \mathrm{ul} \mathrm{FA}$ ). The tubes were vortexed for $5 \mathrm{~min}$ at RT, and then centrifuged for $1 \mathrm{~min}$ at $13,800 \mathrm{~g}$.

\section{Focused-Ultrasonication Method (FUS)}

One to two colonies were added to a microtube containing $80 \mathrm{ul}$ of extraction solution, and then processed in a precooled focused-ultrasonicator (Longlight Technology Co., Ltd, China) under the following conditions: pulse period of 500, pulse width of 250 , running power of 100 , running time of $60 \mathrm{~s}$, and water temperature at $15^{\circ} \mathrm{C}$.

\section{Evaluation of Protein Extraction Methods}

Two novel methods of protein extraction were evaluated in parallel with the routine method. One microliter of supernatant after treatment was transferred to the target plate and allowed to dry at RT before being overlaid with $1 \mathrm{ul}$ of matrix solution (a-cyano-4-hydroxy-cinnamic acid). The acquisition and analysis of mass spectra were performed by VITEK MALDI-TOF MS using the Vitek MS database (MS-ID version v3.2). The results were interpreted referred the manufacturer's instructions. An isolate was considered correctly identified with an acceptable confidence value of $99.9 \%$. Samples were analyzed in duplicates and repeated when there were discrepancies and isolates exhibited discrepant identification results. Results were compared with the sequencing-based identification results and grouped into four categories: a) correct identification: identical to sequencing results, b) incomplete identification (Incomplete ID): either only the genus level was correctly identified or more than one species was proposed and one was correct, c) misidentification (Mis-ID): none of the proposed species were correct, or d) no identification (no-ID).

Spectra were validated with 123 strains by M-Discover 100 MS. The number of peaks and $\mathrm{S} / \mathrm{N}$ ratios were determined by using the program provided by M-Discover. The maximum (or minimum) $\mathrm{S} / \mathrm{N}$ ratio is defined as the height of the highest (or lowest) mass peak above its baseline relative to the standard deviation of the noise.

\section{In-House Database Construction and Clinical Isolates Identification}

According to the evaluation results, we selected the more efficient method to build an in-house library for M-Discover $100 \mathrm{MS}$. One hundred thirty-five isolates were included in the in-house spectra library (Table 2) after following the manufacturer's instructions. Freshly prepared isolates were processed and spotted onto 
TABLE 3 | 467 clinical filamentous fungi isolates identified by M-Discover 100 MS with the in-house library using the ZSB method in comparison with the routine method.

\begin{tabular}{|c|c|c|c|c|c|c|c|c|c|c|c|c|c|c|c|c|c|}
\hline \multirow{3}{*}{$\begin{array}{l}\text { Identification by DNA } \\
\text { sequencing analysis }\end{array}$} & \multirow[t]{3}{*}{ Number } & \multicolumn{8}{|c|}{ ZSB method } & \multicolumn{8}{|c|}{ Routine method } \\
\hline & & \multicolumn{4}{|c|}{$\begin{array}{c}\text { Correct ID to Species } \\
\text { Level }\end{array}$} & \multicolumn{2}{|c|}{$\begin{array}{l}\text { Only Correct ID to Genus } \\
\text { Level }\end{array}$} & \multicolumn{2}{|c|}{ Mis ID } & \multicolumn{4}{|c|}{$\begin{array}{l}\text { Correct ID to Species } \\
\text { Level }\end{array}$} & \multicolumn{2}{|c|}{ Only Correct ID to Genus Level } & \multicolumn{2}{|c|}{ Mis ID } \\
\hline & & Subtotal & $\geq 90$ & $90-60$ & $\leq 60$ & Number & score & Number & score & Subtotal & $\geq 90$ & $90-60$ & $\leq 60$ & Number & score & Number & score \\
\hline \multicolumn{18}{|l|}{ Aspergillus } \\
\hline A. flavus/oryzae & 63 & 63 & 55 & 8 & 0 & 0 & - & 0 & - & 63 & 46 & 15 & 2 & 0 & - & 0 & - \\
\hline A. fumigatus & 184 & 184 & 180 & 4 & 0 & 0 & - & 0 & - & 183 & 179 & 3 & 1 & 1 & - & 0 & - \\
\hline A. lentulus & 2 & 1 & 1 & 0 & 0 & 1 & $\leq 60$ & 0 & - & 1 & 1 & 0 & 0 & 1 & - & 0 & - \\
\hline A. luchuensis & 1 & 1 & 1 & 0 & 0 & 0 & - & 0 & - & 1 & 1 & 0 & 0 & 0 & - & 0 & - \\
\hline A. nidulans & 20 & 20 & 19 & 1 & 0 & 0 & - & 0 & - & 20 & 17 & 3 & 0 & 0 & - & 0 & - \\
\hline A. niger & 58 & 58 & 58 & 0 & 0 & 0 & - & 0 & - & 58 & 53 & 5 & 0 & 0 & - & 0 & - \\
\hline A. sydowii & 1 & 1 & 0 & 1 & 0 & 0 & - & 0 & - & 1 & 0 & 1 & 0 & 0 & - & 0 & - \\
\hline A. terreus & 32 & 32 & 27 & 5 & 0 & 0 & - & 0 & - & 32 & 27 & 5 & 0 & 0 & - & 0 & - \\
\hline A. tubingensis & 36 & 35 & 35 & 0 & 0 & 1 & $\leq 60$ & 0 & - & 35 & 32 & 3 & 0 & 1 & $\leq 60$ & 0 & - \\
\hline A. ustus & 1 & 1 & 1 & 0 & 0 & 0 & - & 0 & - & 1 & 1 & 0 & 0 & 0 & - & 0 & - \\
\hline Subtotal & 398 & 396 & 377 & 19 & 0 & 2 & - & 0 & - & 395 & 357 & 35 & 3 & 3 & - & 0 & - \\
\hline \multicolumn{18}{|l|}{ Fusarium } \\
\hline F. moniliforme & 5 & 4 & 3 & 1 & 0 & 1 & $90-60$ & 0 & - & 4 & 2 & 2 & 0 & 1 & $90-60$ & 0 & - \\
\hline F. proliferatum & 6 & 6 & 0 & 6 & 0 & 0 & - & 0 & - & 4 & 0 & 2 & 2 & 1 & $90-60$ & 1 & $\leq 60$ \\
\hline F. solani & 9 & 7 & 4 & 3 & 0 & 0 & - & 2 & $\leq 60$ & 8 & 0 & 5 & 3 & 0 & - & 1 & $\leq 60$ \\
\hline F. verticillioides & 6 & 0 & 0 & 0 & 0 & 6 & $3(>90) 3(90-60)$ & 0 & - & 0 & 0 & 0 & 0 & 6 & $2(>90) 3(90-60) 1(\leq 60)$ & 0 & - \\
\hline Subtotal & 26 & 17 & 7 & 10 & 0 & 7 & 0 & 2 & & 16 & 2 & 9 & 5 & 8 & - & 2 & - \\
\hline \multicolumn{18}{|l|}{ Penicillium } \\
\hline P. chrysogenum & 1 & 1 & 0 & 0 & 1 & 0 & - & 0 & - & 1 & 0 & 0 & 1 & 0 & - & 0 & - \\
\hline P. citrinum & 1 & 1 & 1 & 0 & 0 & 0 & - & 0 & - & 1 & 1 & 0 & 0 & 0 & - & 0 & - \\
\hline P. oxalicum & 16 & 16 & 16 & 0 & 0 & 0 & - & 0 & - & 16 & 15 & 1 & 0 & 0 & - & 0 & - \\
\hline Subtotal & 18 & 18 & 17 & & 1 & 0 & - & 0 & - & 18 & 16 & 1 & 1 & 0 & - & 0 & - \\
\hline \multicolumn{18}{|l|}{ Scedosporium } \\
\hline S. apiospermum & 2 & 2 & 1 & 1 & 0 & 0 & - & 0 & - & 2 & 1 & 1 & 0 & 0 & - & 0 & - \\
\hline S. aurantiacum & 1 & 1 & 1 & 0 & 0 & 0 & - & 0 & - & 1 & 1 & 0 & 0 & 0 & - & 0 & - \\
\hline S. boydii & 2 & 2 & 2 & 0 & 0 & 0 & - & 0 & - & 2 & 2 & 0 & 0 & 0 & - & 0 & - \\
\hline Subtotal & 5 & 5 & 4 & 1 & 0 & 0 & - & 0 & - & 5 & 4 & 1 & 0 & 0 & - & 0 & - \\
\hline \multicolumn{18}{|l|}{ Others } \\
\hline Alternaria alternata & 3 & 3 & 3 & 0 & 0 & 0 & - & 0 & - & 2 & 0 & 2 & 0 & 0 & - & 1 & $\leq 60$ \\
\hline Beauveria bassiana & 1 & 1 & 0 & 1 & 0 & 0 & - & 0 & - & 0 & 0 & 0 & 0 & 0 & - & 1 & $\leq 60$ \\
\hline Geotrichum candidum & 3 & 3 & 3 & 0 & 0 & 0 & - & 0 & - & 3 & 3 & 0 & 0 & 0 & - & 0 & - \\
\hline Mucor circinelloides & 1 & 1 & 1 & 0 & 0 & 0 & - & 0 & - & 1 & 1 & 0 & 0 & 0 & - & 0 & - \\
\hline Rhizopus oryzae & 2 & 0 & 0 & 0 & 0 & 1 & $\leq 60$ & 1 & $\leq 60$ & 0 & 0 & 0 & 0 & 0 & - & 2 & $\leq 60$ \\
\hline Scopulariopsis brevicaulis & 1 & 1 & 1 & 0 & 0 & 0 & - & 0 & - & 1 & 1 & 0 & 0 & 0 & - & 0 & - \\
\hline Sporothrix schenckii & 1 & 1 & 0 & 0 & 1 & 0 & - & 0 & - & 1 & 0 & 0 & 1 & 0 & - & 0 & - \\
\hline Syncephalastrum racemosum & 2 & 2 & 1 & & 1 & 0 & - & 0 & - & 1 & 1 & 0 & 0 & 0 & - & 1 & $\leq 60$ \\
\hline Trichoderma longibrachiatum & 4 & 4 & 4 & 0 & 0 & 0 & - & 0 & - & 1 & 1 & 0 & 0 & 3 & $90-60$ & & - \\
\hline Trichosporon asahii & 2 & 2 & 1 & 1 & 0 & 0 & - & 0 & - & 2 & 2 & 0 & 0 & 0 & - & 0 & - \\
\hline Subtotal & 20 & 18 & 14 & 2 & 2 & 1 & - & 1 & - & 12 & 9 & 2 & 1 & 3 & - & 5 & - \\
\hline Total & 467 & 454 & 419 & 32 & 3 & 10 & - & 3 & - & 446 & 388 & 48 & 10 & 14 & - & 7 & - \\
\hline
\end{tabular}


eight-well positions on the target plate. Each position was read three times. After excluding the spectra that were obviously abnormal with others, 20 to 24 replica spectra of each strain were added to the in-house library. To verify the accuracy and commonality of the in-house library, the remaining 467 isolates were analyzed. Following the instructions of M-Discover 100 MS, identification scores of $\geq 90$ indicated species-level identification, scores of 60-90 indicated genus-level identification, and scores of $\leq 60$ were considered as "not reliable" (NRI). If isolates exhibited discrepant identification results or produced low matches by M-Discover $100 \mathrm{MS}$ analysis and sequencing analysis, identification by M-Discover $100 \mathrm{MS}$ analysis for the isolates was repeated. In this study, results were compared at the species and genus level with those obtained by sequencing regardless of score values, and grouped into three categories: a) correct identification to species level, b) only correct identification to genus level, c) misidentification.

\section{Statistical Analysis}

Comparison for the identification rates of three protein extraction methods was performed using GraphPad Prism software. This software was also used to compare scores, the peak number, and $\mathrm{S} / \mathrm{N}$ ratios between two groups via a paired t-test. $P<0.05$ indicated a statistically significant difference $\left({ }^{*} p\right.$ values $<0.05$, ${ }^{\star *} p$ values $<0.01,{ }^{* * *} p$ values $<0.005,{ }^{* * *} p$ values $\left.<0.001\right)$.

\section{RESULTS}

\section{Comparison of Identification Performance in VITEK MS}

Table 1 demonstrates the performance of the VITEK MS system for identifying 123 filamentous fungi clinical isolates using the routine and two rapid methods. Among isolates that underwent repeat testing due to discrepancies with the sequencing results, the repeat and original results were consistent. Testing of clinical isolates with two rapid methods revealed significant time savings compared to the routine method. Following the routine procedure, each filamentous fungi isolate required at least $30 \mathrm{~min}$ of sample processing, while the ZSB and FUS method reduced the dedicated operating time to 7 or $5 \mathrm{~min}$ per sample, respectively, with only a few seconds added for each additional strain.

Applying the routine protein extraction method recommended by the manufacturer, VITEK MS correctly identified (species-level identification) 94 (76.42\%) isolates, whereas $98(79.67 \%)$ and 95 (76.42\%) isolates by ZSB and FUS method, respectively. "Incomplete ID" results were produced by the routine and FUS methods for 6 (4.88\%) isolates, and 5 isolates $(4.07 \%)$ by the ZSB method, which all belonged to the Fusarium spp. None of isolates showed a "Mis-ID" result using all methods, while 23 (18.70\%), 20 (16.26\%) and $22(17.89 \%)$ isolates had "no-ID" results by the routine, ZSB, and FUS methods. Of these "no-ID" isolates, 16 isolates belonging to six species were due to no reference spectra available in v3.2 database, except one F. incarnatum that was correctly identified to genus level as " $F$. chlamydosporum complex" by the routine method.

After excluding the isolates that lacked reference spectra, the success rate of identification by VITEK MS applying the routine, ZSB, or FUS method was $92.52 \%$ (99/107) vs 96.26\% (103/107) vs $94.39 \%$ (94/107), and the accuracy was up to $100 \%$ for Aspergillus, Penicillium, Scedosporium, and other spp., except for Fusarium spp. with $72.22 \%$ (13/18), 76.19\% (16/21), and $70.00 \%(14 / 20)$, respectively by three methods. The remaining 8 $(8.75 \%), 4(2.50 \%)$, and $6(6.25 \%)$ isolates had "no-ID", respectively. Upon further analysis, for the Fusarium spp., the VITEK MS showed good ability to identify F. solani $(100 \%$ accuracy rate by three methods), but could not accurately identify $F$. proliferatum and F. verticillioides. The identification ability for $F$. proliferatum and $F$. verticillioides by the ZSB method was $33.33 \%(1 / 6)$ and $83.33 \%(5 / 6)$ with none showing as "no-ID", followed by the FUS method showing $16.67 \%(1 / 6)$ and $66.67 \%(4 / 6)$ with only one F. proliferatum as "no-ID", and $16.67 \%(1 / 6)$ and $33.33 \%(2 / 6)$, with two F. proliferatum and one F. verticillioides showing as "no-ID" by the routine method. VITEK MS was unable to distinguish between the remaining $F$. proliferatum and $F$. verticillioides.

Overall, both ZSB and FUS methods identified isolates from each mold type equal to or better than the routine method without statistically significant differences.

\section{Comparison of Spectral Characteristics in M-Discover 100 MS}

Table $\mathbf{S} 1$ shows the number of peaks per strain by three methods. Overall, the number of peaks performed by the routine method was significantly higher than that of two rapid methods $(P<$ 0.0001). Among the 123 isolates, the peak number of 28 strains extracted by ZSB was more than that extracted by routine method, and 6 strains were the same, mainly distributed in Aspergillus terreus and A. tubingensis. For FUS, 32 strains more than, while 12 strains equal to that of routine method, mainly distributed in A. flavus, A. terreus, and A. tubingensis. And the results of FUS were significantly higher than ZSB $(P=0.0129)$.

The minimum and maximum $\mathrm{S} / \mathrm{N}$ ratios for each isolate are showed in Figure 1. Overall comparing with routine method, significant increase in minimum $\mathrm{S} / \mathrm{N}$ ratios was noted for ZSB $(P<0.0001)$ and FUS $(P=0.0041)$ (Figure 1A). The mean ratio for each species that had two clinical isolates was higher for $16 / 18$ species by ZSB and for $14 / 18$ species by the FUS method, lower than the routine method for A. terreus and $A$. tubingensis by ZSB, and A. flavus, A. lentulus, A. niger, A. terreus by FUS. In addition, statistically higher minimum S/N ratios were obtained for $A$. nidulans, F. solani, $P$. oxalicum, and T. longibrachiatum by both two rapid methods, and Alternaria alternata and S. apiospermum only by ZSB as compared to the routine method.

As observed for the maximum $\mathrm{S} / \mathrm{N}$ ratios, the two rapid method achieved higher overall mean ratio with the 123 isolates $(1,784.1,1,678.4$, and $1,514.4$ for the ZSB, FUS, routine method, respectively). Statistically higher maximum $\mathrm{S} / \mathrm{N}$ ratios were obtained for A. nidulans, A. tubingensis, and P. oxalicum by 
A

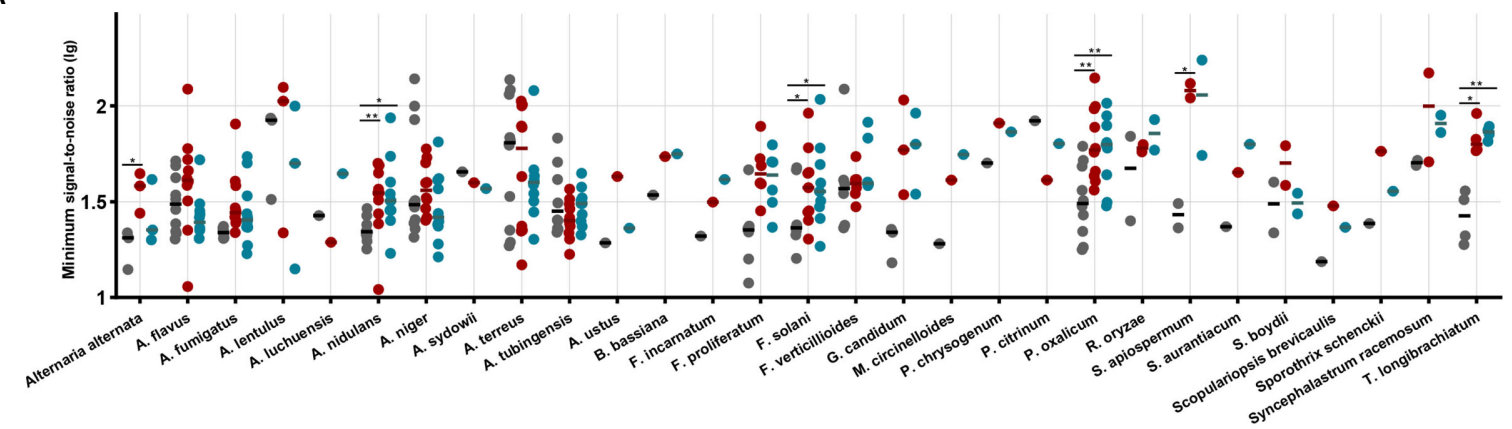

B

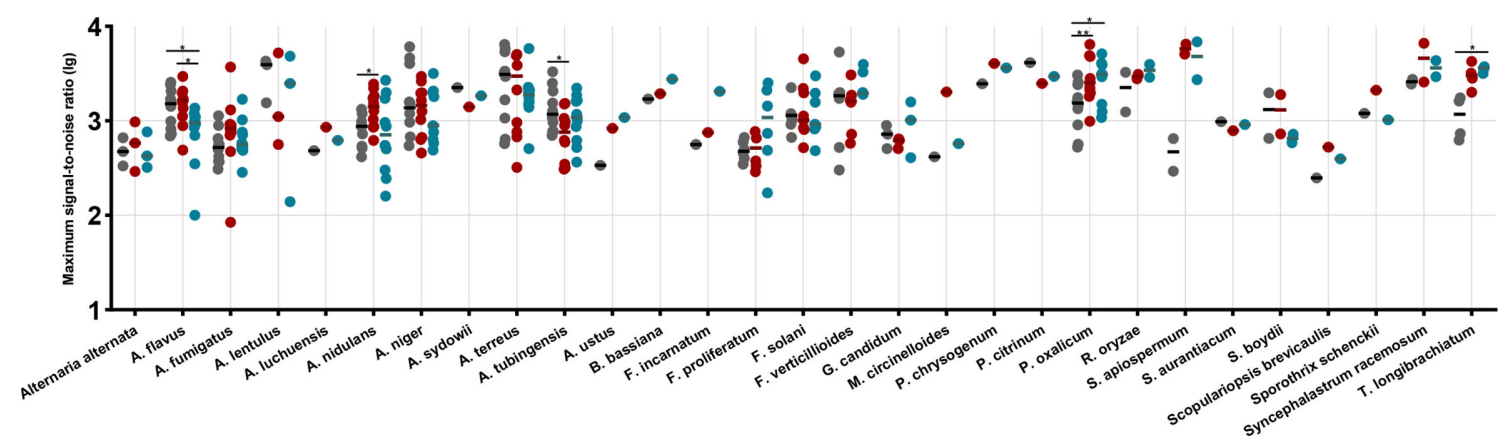

FIGURE 1 | The maximum signal-to-noise ratio (A) and the minimum signal-to-noise ratio (B) by species and by method performed. Each dot represents the signalto-noise ratio (Ig) for each isolate identification achieved by routine (black dots), ZSB (red dots), and FUS (blue dots) methods. Horizontal bars represent the mean ratio $(\mathrm{lg})$ achieved by each method for each species including at least two isolates. A paired t-test was performed to analyze differences between each two groups, and statistical significance was defined by $p$ values less than $0.05\left({ }^{*} p<0.05,{ }^{* *} p<0.01\right)$.

ZSB, while for A. flavus, P. oxalicum, and T. longibrachiatum by FUS as compared to the routine method. The ZSB method produced overall mean ratio higher than that by FUS, and showed superior performance for A. flavus compared to FUS (Figure 1B).

\section{Identification of Filamentous Fungi by In-House Library of M-Discover 100 MS Built by ZSB}

According to the above evaluation results, we selected the ZSB method to build an in-house library for M-Discover $100 \mathrm{MS}$. From the 135 strains distributed by 42 species and 18 genera (Table 2), 2,960 reference spectra were successfully created.

Of the 467 clinical isolates tested, the implementation of the ZSB protein extraction method allowed the correct identification of 99.50\% Aspergillus, 65.38\% Fusarium (absent in the in-house library), 100\% Penicillium/Scedosporium, 90\% other molds, and $454 / 467$ (97.22\%) total clinical isolates by M-Discover $100 \mathrm{MS}$ at the species level using the in-house library regardless of score values (Table 3). Among these 454 isolates, the scores were between 55.76 and 96.90 (median 92.6). A score of $\geq 90$ was obtained for 419 isolates $(92.30 \%$ ), a score of $60-90$ for 32 isolates $(7.05 \%)$, and a score of $\leq 60$ for 3 isolates $(0.66 \%)$. Ten isolates $(2.20 \%)$ were identified at the genus level. All F. verticillioide $(\mathrm{n}=6)$ and one $F$. moniliforme were identified as $F$. moniliforme $(\mathrm{n}=4) / F$. proliferatum $(\mathrm{n}=2$, scores $60-90)$ and F. proliferatum; one A. lentulus, one A. tubingensis, and one Rhizopus oryzae were unreliably identified as A. uvarum (score 40.94), A. niger (score 52.25), and R. baikonurensis (score 43.41). In addition, two $F$. solani and one $R$. oryzae were completely misidentified as Mycobacterium immunogenum/M. malmoense and Nocardia cyriacigeorgicascore (all scores $\leq 60$ ).

In contrast, when the routine method was coupled with the inhouse library, the correct species-level identification rate was $99.74 \% \%, 61.54 \% \%, 100 \%, 60 \%$, and $95.50 \%(n=446$, scores $\geq 90$ for 388 isolates, $60-90$ for 48 isolates, and $\leq 60$ for 10 isolates) for Aspergillus, Fusarium, Penicillium/Scedosporium, other molds, and total isolates, respectively, and $3.00 \%(\mathrm{n}=14)$ to the genus level, thus demonstrating a $1.50 \%(\mathrm{n}=7$, and all with NRI) discrepancy compared to molecular identification. There was no statistically significant difference between the routine and ZSB methods using the in-house library. However, the ability to identify the Fusarium spp. was relatively weak. Among 16/26 (61.54\%) correctly identified Fusarium spp. isolates, only two F. moniliforme were completely identified with a high confidence level (score $\geq 90$ ) and up to five isolates with NRI. In addition, compared to the ZSB method, the routine method was inferior for some rare species, including Alternaria alternata, Beauveria bassiana, Rhizopus oryzae, Syncephalastrum racemosum.

The distribution and mean scores for each species that was isolated at least twice from individual clinical samples are shown in Figure 2. When coupled with the M-Discover 


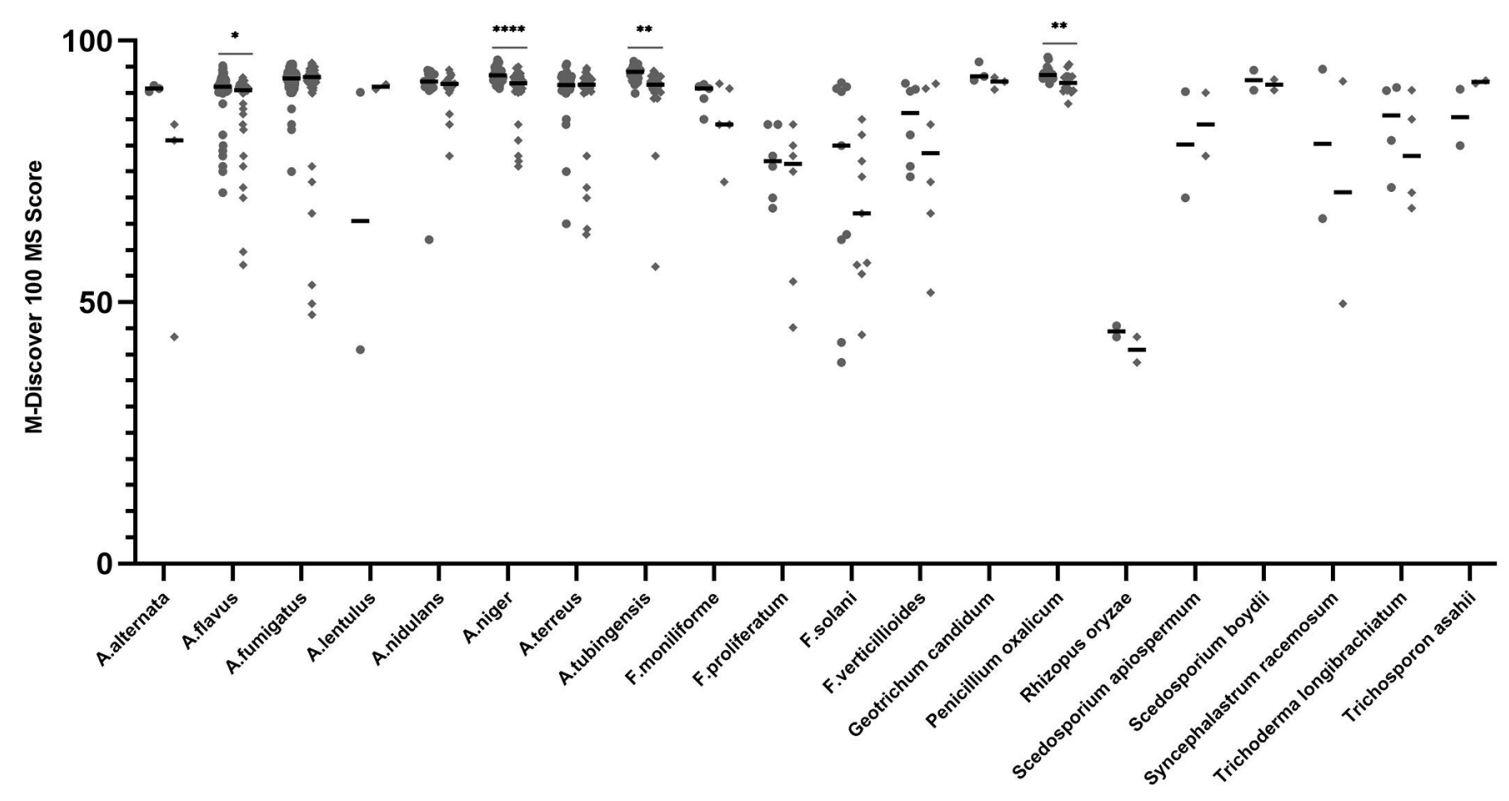

Identified species

FIGURE 2 | Average score and distribution by M-Discover 100 with the in-house library by method performed. Each dot represents the score for each isolate identification achieved by the ZSB (round dots) and routine (diamond dots) methods. Horizontal bars represent the mean identification score achieved by each method for each species including at least two isolates. A paired t-test was performed to analyze differences between the ZSB method and the routine method, and statistical significance was defined by $p$ values less than $0.05\left({ }^{\star} p<0.05,{ }^{* \star} p<0.01,{ }^{* \star \star *} p<0.001\right)$.

100 in-house library, the ZSB method showed comparable or higher maximum and mean scores for most species with the exception of A. lentulus and Scedosporium apiospermum. For the 20 species included, statistically significant differences were only observed in four species (A. flavus, A. niger, A. tubinensis, and $P$. oxalicum). From the point of the score classification, the species-level identification accuracy of strains with the identification score of $\geq 90$ was $99.29 \%$ (419/ 422) provided by ZSB method and $99.49 \%$ (388/390) by the routine method. When the species-level cutoff value was artificially set to 60 , the correct species-level identification rate was $98.47 \%(451 / 458)$ by ZSB and $98.20 \%(436 / 444)$ by routine method.

In addition, the use of the FUS method by the M-Discover 100 system simultaneously using the in-house library also showed good species-assignment of filamentous fungi $(87.00 \%$, $107 / 123)$, which was better than routine method $(84.55 \%, 104 /$ 123), but inferior to the ZSB method (90.24\%) (Table S2).

\section{DISCUSSION}

MALDI-TOF MS has gradually been popularized as an accurate, rapid and cost-effective method for routine identification of clinical filamentous fungi. However, unlike yeast and bacteria, routine clinical use for filamentous fungi is hindered by two main reasons: (i) insufficient filamentous fungi coverage in the commercial database (Schulthess et al., 2014; Li et al., 2017); (ii) prolonged testing time due to the multi-step protein extraction procedure.

To improve work efficiency, we created the one-step zirconiasilica beads method and focused-ultrasonication method for protein extraction. With the routine procedure, each filamentous fungi isolate required at least $30 \mathrm{~min}$ of sample processing before MALDI-TOF MS analysis (Luethy and Zelazny, 2018). The two optimized methods, however, negated the separate inactivation step without reducing the effect (no growth within 14 days after treated), while significantly reduced the dedicated operating time to 7 (ZSB) or 5 (FUS) min per sample, with only a few seconds added for each additional strain.

Of note, using the ZSB and FUS methods with the VITEK MS commercial database v3.2, all of the species resulted in comparable or better identification to the species level than the routine method, except for A. nidulans, A. tubingensis, and Beauveria bassiana. Both rapid methods can be utilized with the existing commercial database v3.2 in VITEK MS, without any required alterations of the database. Similar conclusions have been reported in another study by Luethy and Zelazny (2018). Their study evaluated the capacity of the zirconia-silica beads method combined the high-power bead-based homogenizer for the identification of molds using Bruker MALDI Biotyper, and reported more samples achieving clinically acceptable identification scores $(\geq 2.00)$ than the routine method (63.0 vs 52.8\%) (Adams et al., 2016; Luethy and Zelazny, 2018). Our optimized ZSB method provides significant cost savings in investment (no need for a 
homogenizer) compared to Luethy's method without sacrificing identification effectiveness, while has been verified in two mass spectrometers. In addition, a previous study has proved that the FUS method can significantly increase the identification of mycobacteria by Bruker MALDI Biotyper (Adams et al., 2016). Combining the above two studies and our study, we believe our two rapid methods have universality in most mass spectrometer and species which identification hindered by difficulties related to peptide extraction due to the intrinsic characteristics of the cell. Future studies should be carried out to evaluate and optimize those methods for different mass spectrometers and for more species.

Although VITEK MS reliably identified various filamentous fungi by three methods, including Aspergillus and Penicillium, Scedosporium, and other species with a very low rate of misidentification that was not different from previous reports. For the Fusarium species, which tend to be multi-resistant and are the second most common filamentous fungi causing invasive fungal infections in immunocompromised patients, VITEK MS demonstrated a lower rate of correct identification to the specieslevel by all methods compared than to those in previous studies (100, 93.0, and 65.4\%) (Heo et al., 2015; McMullen et al., 2016; Luethy and Zelazny, 2018; Rychert et al., 2018; Shin et al., 2021).

Given that all of the tested isolates in this study were from northern China, there may be intrinsic differences between the isolates included in the commercial database and those used in this study due to geographic variation (Adams et al., 2016). Specifically, $F$. proliferatum and $F$. verticillioides could not be distinguished by VITEK MS: further examination of the spectra obtained for these clinical isolates of two species revealed the closely related spectra pattern between them. Thus, it is necessary to increase the number of reference isolates in the database in order to distinguish between closely related species well (Lau et al., 2013).

On the other hand, we also evaluated the number of peaks and $\mathrm{S} / \mathrm{N}$ ratios produced by three methods. Since the closed VITEK MS database, the spectra peak cannot be exported, we chose the peaks produced by the M-Discover $100 \mathrm{MS}$ to spectrum analysis. Despite the less number of peaks generated, the two rapid methods generally achieved higher maximum and minimum $\mathrm{S} / \mathrm{N}$ ratios with these isolates tested as compared to the routine method. It is worth noting that not all of these counted peaks are characteristic peaks identified by MS, thus this index may be not a good indicator of the quality of the extraction method. In addition, the ZSB method produced overall mean of maximum and minimum $\mathrm{S} / \mathrm{N}$ ratio higher than that by FUS.

Evaluation of the rapid ZSB method not only revealed good applicability with the existing commercial database, but also demonstrated it can be a rapid and standardized protocol to construct an in-house library. In this study, we used the ZSB method as a protein extraction procedure to construct an inhouse library in M-Discover 100 MS. The inclusion of 2,960 references from 42 species to our in-house database allowed the identification of 454 isolates at the species level (97.22\%) using the ZSB method (Table 3), showing high correlation with DNA sequencing analysis regardless the score values. However,
10 isolates were identified only at the genus level and three completely misidentified, eleven of which were from four different species not available in the in-house database (F. moniliforme, F. verticillioides, F. solani, and Rhizopus oryzae). This highlights the necessity of adding endemic reference strains in the database in order to improve the identification capacity of MALDI-TOF MS. Besides, when lowering the species-level cutoff value to 60 in this study, the correct species-level identification rate showed a high robustness compared with that $(\geq 90)$ in the manufacturer's instructions (98.47 vs 99.29\%). Further research can be done to establish appropriate cutoff value to improve the capacity of MALDI-TOF MS for filamentous fungi.

This in-house library can be used with other protein methods. Although the identification accuracy using the in-house library of both routine and FUS methods were inferior to the ZSB method, there were no statistically significant differences between them. However, the percent of isolates with scores $\geq 90$ and $60-90$ as generated by the routine method was lower than the ZSB method, while statistically significant differences were observed in four species (A. flavus, A. niger, A. tubinensis, and $P$. oxalicum) (Figure 2). There are several possibilities for why fewer accurate identifications occurred. First, it is plausible that the ZSB method breaks the cell wall thoroughly and can achieve higher protein content than the routine method. Further verification, such as comparing protein concentrations and profile are needed according to Akhila's method (Krishnaswamy et al., 2019). Second, the spectra of the same strains had some differences produced by the ZSB and routine method, such as the number and relative position. Thus, the matching degree of the spectra by the routine method with the reference spectra by the ZSB method decreased slightly, resulting in lower scores.

This study has some limitations. First, the strains used in this study represent species typically encountered in northern China. And the Aspergillus species, along with Fusarium and Penicillium species, constitute over half of the tested isolates in this study. Thus, a more comprehensive list of filamentous fungi is needed and filamentous fungi species commonly encountered in other regions should be tested to further evaluate the two rapid methods. Second, the Fusarium species, the second most common strain in this study, was not added to the in-house library, while some common species had only one strain included in the in-house library. It is unknown whether geographic differences of the strains may result in variations in identification accuracy using the inhouse library. Thus, updates to the M-Discover 100 in-house library are necessary to improve the identification ability.

\section{CONCLUSION}

MALDI-TOF MS is widely used for filamentous fungi identification under the condition that an efficient sample processing procedure is implemented and an abundant library is available. Two rapid protein extraction methods we created for filamentous fungi isolates that not only significantly reduced sample processing time but also demonstrated superior 
maximum and minimum $\mathrm{S} / \mathrm{N}$ ratio, and comparable or superior identification to the routine method when utilized with both the existing commercial database and the in-house library. Moreover, to our knowledge, this study represents the first implementation of the zirconia-silica beads method as the sample processing for building an in-house library in MALDITOF MS. We believe the advantages provided by the two rapid methods will attract more clinical laboratories to consider adopting MALDI-TOF MS for filamentous fungi identification.

\section{DATA AVAILABILITY STATEMENT}

The raw data supporting the conclusions of this article will be made available by the authors, without undue reservation.

\section{ETHICS STATEMENT}

The program was approved by the Human Research Ethics Committee of Peking Union Medical College Hospital (S-263).

\section{AUTHOR CONTRIBUTIONS}

Y-TN processed the experimental data, performed the analysis, drafted the manuscript, and revised the manuscript. W-HY, M-X,

\section{REFERENCES}

Adams, L. L., Dionne, K., Fisher, S., and Parrish, N. (2016). A Rapid, Standardized Protein Extraction Method Using Adaptive Focused Acoustics for Identification of Mycobacteria by MALDI-ToF Ms. Diagn. Microbiol. Infect. Dis. 86 (3), 284-288. doi: 10.1016/j.diagmicrobio.2016.06.001

Balajee, S. A., Houbraken, J., Verweij, P. E., Hong, S. B., Yaghuchi, T., Varga, J., et al. (2007). Aspergillus Species Identification in the Clinical Setting. Stud. Mycol. 59, 39-46. doi: 10.3114/sim.2007.59.05

Becker, P. T., de Bel, A., Martiny, D., Ranque, S., Piarroux, R., Cassagne, C., et al. (2014). Identification of Filamentous Fungi Isolates by MALDI-TOF Mass Spectrometry: Clinical Evaluation of an Extended Reference Spectra Library. Med. Mycol. 52 (8), 826-834. doi: 10.1093/mmy/myu064

Benedict, K., Richardson, M., Vallabhaneni, S., Jackson, B. R., and Chiller, T. (2017). Emerging Issues, Challenges, and Changing Epidemiology of Fungal Disease Outbreaks. Lancet Infect. Dis. 17 (12), e403-e411. doi: 10.1016/S14733099(17)30443-7

Bongomin, F., Gago, S., Oladele, R. O., and Denning, D. W. (2017). Global and Multi-National Prevalence of Fungal Diseases-Estimate Precision. J. Fungi (Basel) 3 (4), 57. doi: 10.3390/jof3040057

Brown, G. D., Denning, D. W., Gow, N. A., Levitz, S. M., Netea, M. G., and White, T. C. (2012). Hidden Killers: Human Fungal Infections. Sci. Transl. Med. 4 (165), 113r-165r. doi: 10.1126/scitranslmed.3004404

Cassagne, C., Normand, A. C., L'Ollivier, C., Ranque, S., and Piarroux, R. (2016). Performance of MALDI-TOF MS Platforms for Fungal Identification. Mycoses 59 (11), 678-690. doi: 10.1111/myc.12506

Chakrabarti, A., Bonifaz, A., Gutierrez-Galhardo, M. C., Mochizuki, T., and Li, S. (2015). Global Epidemiology of Sporotrichosis. Med. Mycol. 53 (1), 3-14. doi: $10.1093 / \mathrm{mmy} / \mathrm{myu} 062$

De Carolis, E., Posteraro, B., Lass-Florl, C., Vella, A., Florio, A. R., Torelli, R., et al. (2012). Species Identification of Aspergillus, Fusarium and Mucorales With Direct Surface Analysis by Matrix-Assisted Laser Desorption Ionization Time-
W-Y designed the study and revised the manuscript. W-Z, J-JZ, GZ, and $\mathrm{S}-\mathrm{MD}$ carried out the experiments, performed protein extraction and MALDI-TOF MS identification. A-YD, D-WG, G-LZ, HNW, Y-YG, L-P C, MC, J-DH, and QD contributed to strain collection, performed DNA sequencing identification. LZ and YCX revised the manuscript, and involved in planning and supervised the work. All authors contributed to the article and approved the submitted version.

\section{FUNDING}

This work was supported by the National Major Science and Technology Project for the Control and Prevention of Major Infectious Diseases of China (nos. 2018ZX10712001 and 2020ZX1001015), Beijing Municipal Science and Technology Project (no. Z181100001618015), the National Natural Science Foundation of China (nos. 81802049 and 81971979), and Beijing Key Clinical Specialty for Laboratory Medicine - Excellent Project (no. ZK201000).

\section{SUPPLEMENTARY MATERIAL}

The Supplementary Material for this article can be found online at: https://www.frontiersin.org/articles/10.3389/fcimb.2021. 687240/full\#supplementary-material

of-Flight Mass Spectrometry. Clin. Microbiol. Infect. 18 (5), 475-484. doi: 10.1111/j.1469-0691.2011.03599.x

Doucha, J., and Livansky, K. (2008). Influence of Processing Parameters on Disintegration of Chlorella Cells in Various Types of Homogenizers. Appl. Microbiol. Biotechnol. 81 (3), 431-440. doi: 10.1007/s00253-008-1660-6

Enoch, D. A., Ludlam, H. A., and Brown, N. M. (2006). Invasive Fungal Infections: A Review of Epidemiology and Management Options. J. Med. Microbiol. 55 (Pt 7), 809-818. doi: 10.1099/jmm.0.46548-0

Fraser, M., Brown, Z., Houldsworth, M., Borman, A. M., and Johnson, E. M. (2016). Rapid Identification of 6328 Isolates of Pathogenic Yeasts Using MALDI-ToF MS and a Simplified, Rapid Extraction Procedure That is Compatible With the Bruker Biotyper Platform and Database. Med. Mycol. 54 (1), 80-88. doi: 10.1093/mmy/myv085

Gautier, M., Ranque, S., Normand, A. C., Becker, P., Packeu, A., Cassagne, C., et al. (2014). Matrix-Assisted Laser Desorption Ionization Time-of-Flight Mass Spectrometry: Revolutionizing Clinical Laboratory Diagnosis of Mould Infections. Clin. Microbiol. Infect. 20 (12), 1366-1371. doi: 10.1111/1469-0691.12750

Gilgado, F., Cano, J., Gene, J., Sutton, D. A., and Guarro, J. (2008). Molecular and Phenotypic Data Supporting Distinct Species Statuses for Scedosporium Apiospermum and Pseudallescheria Boydii and the Proposed New Species Scedosporium Dehoogii. J. Clin. Microbiol. 46 (2), 766-771. doi: 10.1128/ JCM.01122-07

Heo, M. S., Shin, J. H., Choi, M. J., Park, Y. J., Lee, H. S., Koo, S. H., et al. (2015). Molecular Identification and Amphotericin B Susceptibility Testing of Clinical Isolates of Aspergillus From 11 Hospitals in Korea. Ann. Lab. Med. 35 (6), 602610. doi: 10.3343/alm.2015.35.6.602

Klimek-Ochab, M., Brzezinska-Rodak, M., Zymanczyk-Duda, E., Lejczak, B., and Kafarski, P. (2011). Comparative Study of Fungal Cell Disruption-Scope and Limitations of the Methods. Folia Microbiol. (Praha) 56 (5), 469-475. doi: 10.1007/s12223-011-0069-2

Kozel, T. R., and Wickes, B. (2014). Fungal Diagnostics. Cold Spring Harb. Perspect. Med. 4 (4), a19299. doi: 10.1101/cshperspect.a019299 
Krishnaswamy, A., Barnes, N., Lotlikar, N. P., and Damare, S. R. (2019). An Improved Method for Protein Extraction From Minuscule Quantities of Fungal Biomass. Indian J. Microbiol. 59 (1), 100-104. doi: 10.1007/s12088018-0752-y

Larone, D. H. (2011). Medically Important Fungi: A Guide to Identification. 5th ed (Washington, DC: ASM Press).

Lau, A. F., Drake, S. K., Calhoun, L. B., Henderson, C. M., and Zelazny, A. M. (2013). Development of a Clinically Comprehensive Database and a Simple Procedure for Identification of Molds From Solid Media by Matrix-Assisted Laser Desorption Ionization-Time of Flight Mass Spectrometry. J. Clin. Microbiol. 51 (3), 828-834. doi: 10.1128/JCM.02852-12

Liao, Y., Chen, M., Hartmann, T., Yang, R. Y., and Liao, W. Q. (2013). Epidemiology of Opportunistic Invasive Fungal Infections in China: Review of Literature. Chin. Med. J. (Engl.) 126 (2), 361-368. doi: 10.3760/ cma.j.issn.0366-6999

Li, S., Plouffe, B. D., Belov, A. M., Ray, S., Wang, X., Murthy, S. K., et al. (2015). An Integrated Platform for Isolation, Processing, and Mass Spectrometry-Based Proteomic Profiling of Rare Cells in Whole Blood. Mol. Cell Proteomics 14 (6), 1672-1683. doi: 10.1074/mcp.M114.045724

Li, Y., Wang, H., Zhao, Y. P., Xu, Y. C., and Hsueh, P. R. (2017). Evaluation of the Bruker Biotyper Matrix-Assisted Laser Desorption/Ionization Time-of-Flight Mass Spectrometry System for Identification of Aspergillus Species Directly From Growth on Solid Agar Media. Front. Microbiol. 8, 1209. doi: 10.3389/ fmicb.2017.01209

Luethy, P. M., and Zelazny, A. M. (2018). Rapid One-Step Extraction Method for the Identification of Molds Using MALDI-TOF Ms. Diagn. Microbiol. Infect. Dis. 91 (2), 130-135. doi: 10.1016/j.diagmicrobio.2018.01.015

McMullen, A. R., Wallace, M. A., Pincus, D. H., Wilkey, K., and Burnham, C. A. (2016). Evaluation of the Vitek Ms Matrix-Assisted Laser Desorption Ionization-Time of Flight Mass Spectrometry System for Identification of Clinically Relevant Filamentous Fungi. J. Clin. Microbiol. 54 (8), 2068-2073. doi: 10.1128/JCM.00825-16

Rodriguez-Sanchez, B., Ruiz-Serrano, M. J., Ruiz, A., Timke, M., Kostrzewa, M., and Bouza, E. (2016). Evaluation of MALDI Biotyper Mycobacteria Library v3.0 for Identification of Nontuberculous Mycobacteria. J. Clin. Microbiol. 54 (4), 1144-1147. doi: 10.1128/JCM.02760-15

Rychert, J., Slechta, E. S., Barker, A. P., Miranda, E., Babady, N. E., Tang, Y. W., et al. (2018). Multicenter Evaluation of the Vitek MS V3.0 System for the Identification of Filamentous Fungi. J. Clin. Microbiol. 56 (2), e01353-17. doi: 10.1128/JCM.01353-17

Samson, R. A., Visagie, C. M., Houbraken, J., Hong, S. B., Hubka, V., Klaassen, C. H., et al. (2014). Phylogeny, Identification and Nomenclature of the Genus Aspergillus. Stud. Mycol. 78, 141-173. doi: 10.1016/j.simyco.2014.07.004

Santos, C. R., Francisco, E., Mazza, M., Padovan, A. C. B., Colombo, A., and Lima, N. (2017). "Impact of MALDI-TOF MS in Clinical Mycology; Progress and Barriers in Diagnostics", in Maldi-TOF and Tandem MS for Clinical Microbiology, 1st ed.
H. N. Shah and S. E. Gharbia, Eds.; (London, UK: John Wiley \& Sons Ltd.) p. 211-230.

Schulthess, B., Ledermann, R., Mouttet, F., Zbinden, A., Bloemberg, G. V., Bottger, E. C., et al. (2014). Use of the Bruker Maldi Biotyper for Identification of Molds in the Clinical Mycology Laboratory. J. Clin. Microbiol. 52 (8), 2797-2803. doi: 10.1128/JCM.00049-14

Seng, P., Drancourt, M., Gouriet, F., La Scola, B., Fournier, P. E., Rolain, J. M., et al. (2009). Ongoing Revolution in Bacteriology: Routine Identification of Bacteria by Matrix-Assisted Laser Desorption Ionization Time-of-Flight Mass Spectrometry. Clin. Infect. Dis. 49 (4), 543-551. doi: 10.1086/600885

Shapaval, V., Moretro, T., Wold, A. A., Suso, H. P., Schmitt, J., Lillehaug, D., et al. (2017). A Novel Library-Independent Approach Based on High-Throughput Cultivation in Bioscreen and Fingerprinting by FTIR Spectroscopy for Microbial Source Tracking in Food Industry. Lett. Appl. Microbiol. 64 (5), 335-342. doi: 10.1111/lam.12691

Shin, J. H., Kim, S. H., Lee, D., Lee, S. Y., Chun, S., Lee, J. H., et al. (2021). Performance Evaluation of VITEK MS for the Identification of a Wide Spectrum of Clinically Relevant Filamentous Fungi Using a Korean Collection. Ann. Lab. Med. 41 (2), 214-220. doi: 10.3343/alm.2021.41.2.214

Wang, H., Xiao, M., Kong, F., Chen, S., Dou, H. T., Sorrell, T., et al. (2011). Accurate and Practical Identification of 20 Fusarium Species by Seven-Locus Sequence Analysis and Reverse Line Blot Hybridization, and an In Vitro Antifungal Susceptibility Study. J. Clin. Microbiol. 49 (5), 1890-1898. doi: 10.1128/JCM.02415-10

Welham, K. J., Domin, M. A., Johnson, K., Jones, L., and Ashton, D. S. (2000). Characterization of Fungal Spores by Laser Desorption/Ionization Time-ofFlight Mass Spectrometry. Rapid Commun. Mass Spectrom. 14 (5), 307-310. doi: 10.1002/(SICI)1097-0231(20000315)14:5<307::AID-RCM823>3.0.CO;2-3

Wickes, B. L., and Wiederhold, N. P. (2018). Molecular Diagnostics in Medical Mycology. Nat. Commun. 9 (1), 5135. doi: 10.1038/s41467-018-07556-5

Zvezdanova, M. E., Escribano, P., Ruiz, A., Martinez-Jimenez, M. C., Pelaez, T., Collazos, A., et al. (2019). Increased Species-Assignment of Filamentous Fungi Using MALDI-TOF MS Coupled With a Simplified Sample Processing and an in-House Library. Med. Mycol. 57 (1), 63-70. doi: 10.1093/mmy/myx154

Conflict of Interest: The authors declare that the research was conducted in the absence of any commercial or financial relationships that could be construed as a potential conflict of interest.

Copyright (C) 2021 Ning, Yang, Zhang, Xiao, Wang, Zhang, Zhang, Duan, Dong, Guo, Zou, Wen, Guo, Chen, Chai, He, Duan, Zhang, Zhang and Xu. This is an open-access article distributed under the terms of the Creative Commons Attribution License (CC BY). The use, distribution or reproduction in other forums is permitted, provided the original author(s) and the copyright owner(s) are credited and that the original publication in this journal is cited, in accordance with accepted academic practice. No use, distribution or reproduction is permitted which does not comply with these terms. 